\title{
Methods underpinning national clinical guidelines for hypertension: describing the evidence shortfall
} Fiona Campbell ${ }^{1}$, Heather O Dickinson ${ }^{1}$, Julia VF Cook ${ }^{1}$, Fiona R Beyer ${ }^{1}$, Martin Eccles ${ }^{1}$ and James M Mason*2

\author{
Address: ${ }^{1}$ University of Newcastle upon Tyne, Centre for Health Services Research, 21 Claremont Place, Newcastle upon Tyne, NE2 4AA, UK and \\ ${ }^{2}$ University of Durham, School for Health, Wolfson Research Institute, Queen's Campus, University Boulevard, Stockton-on-Tees, TS17 6BH, UK \\ Email: Fiona Campbell - fiona.campbell@ncl.ac.uk; Heather O Dickinson - heather.dickinson@ncl.ac.uk; Julia VF Cook - j.v.f.cook@ncl.ac.uk; \\ Fiona R Beyer - fiona.beyer@ncl.ac.uk; Martin Eccles - martin.eccles@ncl.ac.uk; James M Mason* - j.m.mason@durham.ac.uk \\ * Corresponding author
}

Published: 05 April 2006

BMC Health Services Research 2006, 6:47 doi:10.1186/1472-6963-6-47

This article is available from: http://www.biomedcentral.com/I472-6963/6/47

(c) 2006 Campbell et al; licensee BioMed Central Ltd.

This is an Open Access article distributed under the terms of the Creative Commons Attribution License (http://creativecommons.org/licenses/by/2.0), which permits unrestricted use, distribution, and reproduction in any medium, provided the original work is properly cited.
Received: 31 August 2005

Accepted: 05 April 2006

\begin{abstract}
Background: To be useful, clinical practice guidelines need to be evidence based; otherwise they will not achieve the validity, reliability and credibility required for implementation.

Methods: This paper compares the methods used in gathering, analysing and linking of evidence to guideline recommendations in ten current hypertension guidelines.

Results: It found several guidelines had failed to implement methods of searching for the relevant literature, critical analysis and linking to recommendations that minimise the risk of bias in the interpretation of research evidence. The more rigorous guidelines showed discrepancies in recommendations and grading that reflected different approaches to the use of evidence in guideline development.
\end{abstract}

Conclusion: Clinical practice guidelines as a methodology are clearly still an evolving health care technology.

\section{Background}

Clinical practice guidelines can provide building blocks for changing and improving health care [1] and are a useful means of bridging the gap between scientific research evidence and usual practice [2]. They are defined as 'systematically developed statements to assist physicians and patients about appropriate health care for specific clinical circumstances' [3]. To achieve their potential as effective tools for improving health care they need to maximise their validity, a feature related to the use of evidence within a guideline and development using a multidisciplinary process [4]. However, despite an apparently explicit methodology there are variations in what guidelines say and how they relate this to underlying evidence $[3,5,6]$. There is also concern that guideline development may be subject to external influence $[7,8]$.

Like many other conditions hypertension has been the subject of many different international guidelines. The World Health Organisation (WHO) have described hypertension - defined as a blood pressure of greater than $140 / 90 \mathrm{mmHg}$ - as one of the ten leading risk factors influencing the global burden of disease [9]. It is a contributory factor in ischaemic heart disease and cerebrovascular disease accounting for $20 \%$ and $10 \%$ of all deaths in England and Wales respectively [10]. Reducing blood 
Table I: Recent major guidelines for hypertension

\begin{tabular}{lcc}
\hline Organisation & Publication Year & Acronym \\
\hline Canadian Medical Association [13] & 1999 & CMA \\
World Health Organisation - International & 1999 & WHO \\
Society of Hypertension [14] & 2000 & VHA \\
Veterans Health Administration (US) [15] & 2001 & SIGN \\
Scottish Intercollegiate Guidelines Network & & ESH \\
[I8] & 2003 & ICSI \\
European Society of Hypertension[19] & 2003 & JNC \\
Institute for Clinical Systems Improvement & & SA \\
(US) [16] Joint National Committee VII (US) & 2003 & BHS \\
Southern African Hypertension Society [17] & 2003 & NICE \\
British Hypertension Society [2I] & 2004 & 2004 \\
National Institute for Clinical Excellence [22] & & \\
\hline
\end{tabular}

pressure levels leads to significant reductions in cardiovascular and cerebrovascular morbidity and mortality [11]. Nevertheless the management of hypertension remains suboptimal: for example, $40 \%$ of the adult population of England suffer from hypertension, but current levels of detection and treatment result in only $9 \%$ of sufferers having their hypertension controlled to $<140 / 90 \mathrm{~mm} \mathrm{Hg}$ [12]. There is a clear need to improve the management of hypertension both in this country and worldwide. If guidelines, however, are to play a role in this improvement they will need to maximise their validity.

The aim of this study was to review how well 10 guidelines for hypertension addressed validity in terms of their methods and their use of published evidence.

\section{Methods \\ Contributing guidelines}

We reviewed the methods used in development and the key recommendations of ten current guidelines (see table 1) meeting the following criteria: they concerned the general management of hypertension, or the management of hypertension in specific populations; published in English and nationally or internationally recognised. Guidelines developed before 1994 were also excluded as they predated the publication and wide dissemination of the work by Field and Lohr which offered the first and seminal work on guideline methodology [1].

We used five guidelines (CMA [13], WHO [14], VHA [15], ICSI [16], SA [17]) meeting these criteria retrieved by the comprehensive search strategy employed by the German Guideline Clearing Report [7], whose search strategy cov-

Table 2: Methodological aspects of recent major guidelines for hypertension

\begin{tabular}{|c|c|c|c|c|c|c|}
\hline Guideline & $\begin{array}{c}\text { Development } \\
\text { group } \\
\text { described }^{a}\end{array}$ & $\begin{array}{l}\text { New systematic } \\
\text { reviewing }\end{array}$ & $\begin{array}{l}\text { Used existing } \\
\text { systematic } \\
\text { reviews }\end{array}$ & $\begin{array}{l}\text { Literature } \\
\text { search }^{\mathbf{b}}\end{array}$ & $\begin{array}{l}\text { Grading of evi- } \\
\text { dence }^{d}\end{array}$ & $\begin{array}{l}\text { Grading of rec- } \\
\text { ommendations }^{\text {d }}\end{array}$ \\
\hline CMA & $P$ & $\mathrm{~N}$ & $Y$ & $Y$ & $Y$ & $Y$ \\
\hline WHO & $P$ & $\mathrm{~N}$ & $Y$ & $\mathrm{~N}$ & $\mathrm{~N}$ & $\mathrm{~N}$ \\
\hline VHA & $P$ & $N$ & $N$ & $Y$ & $Y$ & $Y$ \\
\hline SIGN & $Y$ & $N$ & $Y$ & $Y$ & $Y$ & $Y$ \\
\hline $\mathrm{ESH}$ & $Y$ & $N$ & $Y$ & $\mathrm{~N}$ & $\mathrm{~N}$ & $N$ \\
\hline ICSI & $\mathrm{N}$ & $\mathrm{N}$ & $y$ & $\mathrm{~N}$ & $Y$ & $\mathrm{~N}$ \\
\hline JNC & $P$ & $\mathrm{~N}$ & $Y$ & $N$ & $Y$ & $N$ \\
\hline SA & $P$ & $N$ & $y$ & $N$ & $\mathrm{~N}$ & $N$ \\
\hline $\mathrm{BHS}$ & $P$ & $\mathrm{~N}$ & $Y$ & $\mathrm{~N}$ & $Y$ & $Y$ \\
\hline NICE & $Y$ & $Y$ & $Y$ & $Y$ & $Y$ & $Y$ \\
\hline
\end{tabular}

a $\mathrm{Y} / \mathrm{N}$ : Includes/does not include description of stakeholders involved in the development process, including patient representatives and any conflicts of interest.

P: Partially - a list of names with institutional affiliations was provided.

b Y/N: Searches were/were not undertaken, at least through Medline.

c Y: Made extensive use of existing systematic reviews (referenced 7 or more).

y: Made partial use of existing systematic reviews (referenced 6 or less).

$\mathrm{N}$ : Did not use existing systematic reviews.

$d Y / N$ : Reports/does not report explicit grading of the quality of the supporting evidence/recommendations. 
Table 3: Methodological aspects of previously published guidelines

\begin{tabular}{|c|c|c|c|}
\hline Guideline & Literature search & Grading of evidence & Development group \\
\hline CMA & $\begin{array}{l}\text { MEDLINE and Cochrane } \\
\text { Collaboration searches; reference } \\
\text { lists in retrieved articles. Requests } \\
\text { to experts and panel members. }\end{array}$ & $\begin{array}{l}\text { Recommendations were graded } \\
\text { from A-D and were based on } \\
\text { assessment of the studies using an } \\
\text { algorithm. } \\
\text { A Grade assigned if } \\
\text { recommendation was: } \\
\text { - based on an adequate RCT ie, } \\
\text { with blinded assessment of } \\
\text { outcomes, intention-to-treat } \\
\text { analysis, adequate follow-up, and } \\
\text { sufficient sample size to detect a } \\
\text { clinically important difference with } \\
\text { power greater than } 80 \% \text {. } \\
\text { - based on an adequate subgroup } \\
\text { analysis } \\
\text { - based on a systematic review in } \\
\text { which the comparison arms are } \\
\text { derived from head-to head } \\
\text { comparisons with the same RCT }\end{array}$ & $\begin{array}{l}\text { A committee with a range of } \\
\text { representatives from different } \\
\text { bodies. Patient involvement unclear }\end{array}$ \\
\hline WHO & None described & None described & Patient involvement unclear \\
\hline VHA & $\begin{array}{l}\text { MeSH terms covering key therapies, } \\
\text { and study characteristics and design }\end{array}$ & $\begin{array}{l}\text { Evidence was graded: } \\
\text { A RCT } \\
\text { B well-designed clinical studies } \\
\text { C panel consensus } \\
\text { Recommendations were rated: } \\
\text { I usually indicated; always } \\
\text { acceptable; useful and effective } \\
\text { lla acceptable, of uncertain } \\
\text { effectiveness, and may be } \\
\text { controversial. Weight of evidence } \\
\text { in favour of usefulness/effectiveness } \\
\text { Ilb acceptable, of uncertain } \\
\text { effectiveness and may be } \\
\text { controversial. Not well established } \\
\text { by evidence, can be helpful and } \\
\text { probably not harmful }\end{array}$ & $\begin{array}{l}\text { Thirty-eight individuals. Roles not } \\
\text { always clear. }\end{array}$ \\
\hline SIGN & $\begin{array}{l}\text { Systematic literature searches on } \\
\text { MEDLINE, Healthstar, EMBASE, } \\
\text { Cochrane Library. Based on a } \\
\text { published Cochrane review }\end{array}$ & $\begin{array}{l}\text { Evidence was graded when obtained } \\
\text { from: } \\
\text { la: meta-analysis of RCTs lb: at least } \\
\text { I RCT } \\
\text { Ila: at least I well-designed } \\
\text { controlled study without } \\
\text { randomisation } \\
\text { Ilb: at least one other type of well- } \\
\text { designed quasi-experimental study } \\
\text { III: well-designed non-experimental } \\
\text { descriptive studies } \\
\text { IV: expert committee reports and/ } \\
\text { or respected clinical opinion } \\
\text { Recommendations were rated: } \\
\text { A Evidence levels la, Ib } \\
\text { B Evidence levels Ila, IIb, III } \\
\text { C Evidence level IV. }\end{array}$ & $\begin{array}{l}\text { Members' names and affiliations } \\
\text { listed and conflicts of interest } \\
\text { available. Specialist reviewer names } \\
\text { given. Age Concern represented. }\end{array}$ \\
\hline ESH & None described & $\begin{array}{l}\text { Recommendations not classified } \\
\text { upon strength of available evidence. }\end{array}$ & $\begin{array}{l}\text { Members' names, affiliations, } \\
\text { potential conflicts of interest given. } \\
\text { Patient involvement unclear }\end{array}$ \\
\hline
\end{tabular}


Table 3: Methodological aspects of previously published guidelines (Continued)

\begin{tabular}{|c|c|c|c|}
\hline ICSI & None described & $\begin{array}{l}\text { Research reports were graded as } \\
\text { follows: } \\
\text { Primary reports A (RCT) to D } \\
\text { (case and cross sectional studies) } \\
\text { Reviews M (Meta-analysis, } \\
\text { systematic reviews, decision } \\
\text { analysis, cost-benefit analysis, cost- } \\
\text { effectiveness study) } \\
\text { R: Narrative review, consensus } \\
\text { statement or report } \\
X: \text { Medical opinion } \\
\text { In the } 2002 \text { update, some } \\
\text { recommendations link to the } \\
\text { evidence grade }\end{array}$ & No details \\
\hline JNC & None described & $\begin{array}{l}\text { Evidence supporting } \\
\text { recommendations for prevention } \\
\text { and treatment was classified: } \\
\text { M meta-analysis } \\
\text { Re retrospective analyses (case } \\
\text { control) } \\
\text { RA RCT } \\
\text { F prospective follow-up - cohort } \\
\text { study } \\
\text { Pr previous review } \\
\text { C clinical interventions (non- } \\
\text { randomised) } \\
\text { X cross-sectional population } \\
\text { studies (prevalence) }\end{array}$ & $\begin{array}{l}\text { Nine individuals. Contributions } \\
\text { were sought from multidisciplinary } \\
\text { experts. No mention of patient } \\
\text { involvement }\end{array}$ \\
\hline SA & None described & Evidence not described or graded & $\begin{array}{l}\text { Members' names and affiliation } \\
\text { given. Patient involvement unclear }\end{array}$ \\
\hline BHS & Not described & $\begin{array}{l}\text { Strength of evidence: la (meta } \\
\text { analysis of RCTs) to IV expert } \\
\text { opinion Strength of } \\
\text { recommendation } \\
\text { A Directly based on category I } \\
\text { evidence } \\
\text { B Directly based on category II } \\
\text { evidence or extrapolated } \\
\text { recommendation from category I } \\
\text { evidence } \\
\text { C Directly based on category III } \\
\text { evidence or extrapolated } \\
\text { recommendation from category I or } \\
\text { II evidence. } \\
\text { D Directly based on category IV } \\
\text { evidence or extrapolated } \\
\text { recommendation from category I, II } \\
\text { or III evidence }\end{array}$ & No details given \\
\hline NICE & $\begin{array}{l}\text { Search using MEDLINE, EMBASE } \\
\text { and CENTRAL, previous systematic } \\
\text { reviews, bibliographic seachs as well } \\
\text { as contact with subject area } \\
\text { experts. }\end{array}$ & $\begin{array}{l}\text { Guideline Recommendation and } \\
\text { Evidence Grading (GREG [34]) } \\
\text { system applied. } \\
\text { Evidence Grade: Interpretation } \\
\text { of Evidence } \\
\text { I High Plausible, precisely quantified } \\
\text { and not vulnerable to bias. } \\
\text { II Intermediate Plausible but not } \\
\text { quantified precisely or may be } \\
\text { vulnerable to bias. } \\
\text { III Low Concerns about plausibility } \\
\text { or vulnerability to bias. } \\
\text { Recommendation Grade } \\
\text { Interpretation of } \\
\text { recommendation } \\
\text { A Recommendation Robust } \\
\text { evidence } \\
\text { B Provisional Recommendation } \\
\text { Recommend with caution } \\
\text { C Consensus Opinion } \\
\text { Recommended by consensus }\end{array}$ & $\begin{array}{l}\text { Members names and affiliations } \\
\text { given and any potential conflicts of } \\
\text { interest. Contributions were sought } \\
\text { from multidisciplinary experts. } \\
\text { Group included patient } \\
\text { representatives }\end{array}$ \\
\hline
\end{tabular}


ered 1990-1999. We updated the strategy by searching MEDLINE, EMBASE and OMNI from 1999 onwards using the thesaurus heading HYPERTENSION and limiting to guidelines or practice guidelines in English. This retrieved a further five guidelines (SIGN [18], ESH [19], JNC [20], BHS [21], NICE [22]) for consideration.

\section{Evaluation of guideline development methods}

We evaluated the methods used to develop each guideline with particular reference to three dimensions that relate to the use of research evidence, as found in the full published report of each guideline:

- the construction of the guideline development group and its component stakeholders.

- the use of published literature and the strategy used in screening for the primary evidence; in particular, the use of existing systematic reviews or the performance of a new systematic review explicitly to answer questions posed by the guideline.

- the grading of evidence and recommendations: in particular, an explicit link between recommendations and supporting evidence.

\section{Evaluation of recommendations and their underlying evidence}

We compared recommendations on four areas that were common to all the guidelines: diagnosis of hypertension, lifestyle modification, criteria for initiation of antihypertensive drug therapies and initial recommended drug therapy. We also explored links between recommendation grades and citations and looked at how these differed in recommendations for drug therapy and salt intake.

\section{Results}

\section{Methods used to develop the guidelines}

The measures used to assess the guideline development process are summarised in table 2 .

Only three guidelines were constructed by multidisciplinary groups where the members' affiliations and conflicts of interest were described; these three guideline groups included patient representatives as well as key professional stakeholders. A further six guidelines provided only a list of names and institutional affiliations of members of the guideline development group. One further guideline gave no details of the guideline development group (see table 3).

Only one guideline conducted new systematic reviews to inform recommendations. Seven guidelines made extensive use of existing systematic reviews; three of these guidelines also stated that a search strategy based on
MESH search terms was used for identifying relevant research evidence. A further two guidelines made limited use of existing systematic reviews.

There were different approaches used in the guidelines to assess the evidence available upon which to base a recommendation and upon the grading of the recommendation itself (see table 3 for a description of the grading systems used in the guidelines). Three guidelines did not grade either the evidence they cited or their recommendations. Two coded the evidence on the basis of study design but did not link this to the recommendations. In contrast, five guidelines graded the evidence and explicitly linked this to the recommendations. However, there were differences also between these grading systems with different criteria used to assess the contributing studies. The grading systems used by two guidelines (NICE, CMA) allowed for the quality of relevant randomised controlled trials and metaanalyses and the strength of their results to be analysed. The grading systems used by other guidelines did not allow for this more sensitive assessment of the evidence. Differences and shortcomings in these grading systems can be confusing and impede effective communication [23]. The GRADE system was developed as a result of these shortcomings and recommends an approach which takes into account study design, quality, consistency and directness in judging the considers the benefit harm ratio, quality of evidence, applicability, and baseline risk when translating to recommendation [23].

\section{Recommendations made by the guidelines Diagnosis of hypertension}

The guidelines were consistent in defining the threshold for hypertension as 140/90 $\mathrm{mmHg}$ and all agreed that blood pressure should be measured twice in a consultation on at least two separate occasions. The need for a full medical examination, clinical history and accurate blood pressure measurement was described in all of the guidelines. However, they differed on the recommended routine tests. All agreed that an electrocardiogram, blood chemistry, a complete blood cell count and urinalysis should be conducted during the initial assessment of hypertension, to assess broader cardiovascular risk. There was less agreement on the assessment of: total cholesterol, lipid profile, blood glucose, creatinine, blood calcium, thyroid stimulating hormone, gammaglutamyl transpeptidase and serum urate.

\section{Lifestyle modifications}

All of the guidelines addressed lifestyle modification as an integral part of the management of hypertension and as a first line treatment in mild hypertension, and made similar recommendations for weight loss, limiting alcohol and sodium intake, regular exercise and smoking cessation (see table 4). Guidelines typically recommended a target 


\begin{tabular}{|c|c|c|c|c|c|c|c|c|}
\hline \multirow[t]{2}{*}{ Guideline } & \multirow{2}{*}{$\begin{array}{l}\text { Weight reduction } \\
\text { Recommended weight }\end{array}$} & \multicolumn{3}{|l|}{ Diet } & \multirow{2}{*}{$\begin{array}{l}\text { Salt restriction } \\
\text { Recommended max. } \\
\text { daily intake }\end{array}$} & \multirow{2}{*}{$\begin{array}{l}\text { Alcohol restriction } \\
\text { Recommended max. } \\
\text { daily intake (ethanol) }\end{array}$} & \multirow{2}{*}{$\begin{array}{l}\text { Smoking } \\
\text { cessation }\end{array}$} & \multirow[t]{2}{*}{ Exercise } \\
\hline & & $\begin{array}{l}\text { Reduce saturated } \\
\text { fat }\end{array}$ & $\begin{array}{l}\text { Increase fruit \& } \\
\text { vegetables }\end{array}$ & $\begin{array}{l}\text { Other } \\
\text { recommendations }\end{array}$ & & & & \\
\hline CMA & $\begin{array}{l}\text { Y } \\
\left(\text { BMI } 20-25 \mathrm{~kg} / \mathrm{m}^{2}\right)\end{array}$ & \multicolumn{3}{|c|}{ No specific recommendation } & $\begin{array}{l}Y \\
(3-7 \text { g/day })\end{array}$ & $\begin{array}{l}\leq 17.4 \mathrm{~g} / \text { day (women) } \\
\leq 27.0 \mathrm{~g} / \text { day (men) }\end{array}$ & Y & $\begin{array}{l}50-60 \text { mins. aerobic } \\
\text { exercise } 3-4 \text { times per } \\
\text { week }\end{array}$ \\
\hline WHO & $Y$ if overweight & $Y$ & $\mathrm{Y}$ & $\begin{array}{l}\text { Increase fish } \\
\text { consumption }\end{array}$ & $\begin{array}{l}Y \\
(\leq 6 \mathrm{~g} / \text { day })\end{array}$ & $\begin{array}{l}\leq 10-20 \mathrm{~g} / \text { day (women) } \\
\leq 20-30 \mathrm{~g} / \text { day (men) }\end{array}$ & $Y$ & $\begin{array}{l}30-45 \text { mins. aerobic } \\
\text { exercise } 3-4 \text { times / } \\
\text { week }\end{array}$ \\
\hline VHA & $\begin{array}{l}Y \\
\text { (if }>10 \% \text { of ideal body weight) }\end{array}$ & $Y$ & Y & $\begin{array}{l}\text { Increase cereal } \\
\text { consumption }\end{array}$ & $\begin{array}{l}Y \\
(\leq 6 \mathrm{~g} / \text { day })\end{array}$ & $\begin{array}{l}\leq 14 \mathrm{~g} / \text { day (women) } \\
\leq 28 \mathrm{~g} / \text { day (men) }\end{array}$ & $Y$ & $\begin{array}{l}30-45 \text { mins. aerobic } \\
\text { exercise } 3-5 \text { times/ } \\
\text { week }\end{array}$ \\
\hline SIGN & $\begin{array}{l}\text { Y } \\
\left(\mathrm{BMI}<25 \mathrm{~kg} / \mathrm{m}^{2}\right)\end{array}$ & Y & Y & & $\begin{array}{l}Y \\
(<5 \mathrm{~g} / \text { day })\end{array}$ & $\begin{array}{l}\leq 15.8 \mathrm{~g} / \text { day }(\text { women }) \\
\leq 23.7 \mathrm{~g} / \text { day }(\text { men })\end{array}$ & Y & $\begin{array}{l}30-45 \text { mins. aerobic } \\
\text { exercise most days }\end{array}$ \\
\hline ESH & $\mathrm{Y}$ & $\mathrm{Y}$ & Y & $\begin{array}{l}\text { Increase fish } \\
\text { consumption }\end{array}$ & $\begin{array}{l}Y \\
(4.7-5.8 \mathrm{~g} / \text { day })\end{array}$ & $\begin{array}{l}\leq 10-20 \mathrm{~g} / \text { day (women) } \\
\leq 20-30 \mathrm{~g} / \text { day (men) }\end{array}$ & $\mathrm{Y}$ & $\begin{array}{l}30-45 \text { mins. aerobic } \\
\text { exercise 3-5 times/ } \\
\text { week }\end{array}$ \\
\hline ICSI & $Y$ if overweight & $Y$ & Y & $\begin{array}{l}\text { Increase low fat } \\
\text { dairy food } \\
\text { consumption }\end{array}$ & $\begin{array}{l}Y \\
(<6 \text { g/day })\end{array}$ & $\begin{array}{l}\leq 14 \text { g/day (women) } \\
\leq 28 \text { g/day (men) }\end{array}$ & $Y$ & $\begin{array}{l}30-45 \text { mins. aerobic } \\
\text { exercise } 3-4 \text { times/ } \\
\text { week }\end{array}$ \\
\hline JNC & $\begin{array}{l}\text { Y } \\
\left(\text { BMI } 18.5-25 \mathrm{~kg} / \mathrm{m}^{2}\right)\end{array}$ & Y & Y & $\begin{array}{l}\text { Increase low fat } \\
\text { dairy food } \\
\text { consumption }\end{array}$ & $\begin{array}{l}Y \\
(\leq 6 \mathrm{~g} / \text { day })\end{array}$ & $\begin{array}{l}\leq 14 \mathrm{~g} / \text { day (women) } \\
\leq 28 \mathrm{~g} / \text { day (men) }\end{array}$ & $\begin{array}{l}\text { Not } \\
\text { discussed }\end{array}$ & $\begin{array}{l}\geq 30 \text { mins. aerobic } \\
\text { exercise most days }\end{array}$ \\
\hline SA & $\begin{array}{l}\text { Y } \\
\left(\mathrm{BMI}<25 \mathrm{~kg} / \mathrm{m}^{2}\right)\end{array}$ & Y & Y & $\begin{array}{l}\text { Increase fibre, } \\
\text { unrefined } \\
\text { carbohydrates }\end{array}$ & Y & $\leq 24$ g/day & $Y$ & $\begin{array}{l}30 \text { mins. aerobic } \\
\text { exercise } 3-5 \text { times per } \\
\text { week }\end{array}$ \\
\hline BHS & $\begin{array}{l}\text { Y } \\
\left(\text { BMI } 20-25 \mathrm{~kg} / \mathrm{m}^{2}\right)\end{array}$ & Y & Y & Reduce total fat & $\begin{array}{l}Y \\
(<6 \text { g/day })\end{array}$ & $\begin{array}{l}\leq 15.8 \mathrm{~g} / \text { day } \\
\leq 23.7 \mathrm{~g} / \text { day (men) }\end{array}$ & Y & $\begin{array}{l}>30 \text { mins. aerobic } \\
\text { exercise most days }\end{array}$ \\
\hline NICE & $Y$ & Healthy, low calo & e diet & & $\begin{array}{l}Y \\
(<6 \text { g/day })\end{array}$ & $\begin{array}{l}<14 \text { units/wk (women) } \\
<21 \text { units/week (men) }\end{array}$ & $Y$ & $\begin{array}{l}30-60 \text { mins. 3-5 times/ } \\
\text { week }\end{array}$ \\
\hline
\end{tabular}


Table 5: Recommended thresholds for drug treatment and initial drug therapy in recent major guidelines for hypertension

\begin{tabular}{|c|c|c|c|c|c|c|}
\hline \multirow[b]{2}{*}{ Guideline } & \multicolumn{4}{|c|}{ Thresholds for initiating drug treatment* } & \multicolumn{2}{|c|}{$\begin{array}{l}\text { Initial drug therapy in } \\
\text { uncomplicated patient (non-black } \\
\text { patient aged over } 55-60 \text { years) } \dagger\end{array}$} \\
\hline & $\begin{array}{l}\text { No target organ } \\
\text { damage or risk } \\
\text { factors }\end{array}$ & $\begin{array}{l}\text { With risk factors } \\
\text { (other than diabetes } \\
\text { mellitus) }\end{array}$ & $\begin{array}{l}\text { With target organ } \\
\text { damage }\end{array}$ & $\begin{array}{l}\text { With diabetes } \\
\text { mellitus or renal } \\
\text { disease }\end{array}$ & Recommendation & $\begin{array}{l}\text { Grade of } \\
\text { recommendation }\end{array}$ \\
\hline CMA & $\geq 160 / 90$ & $\geq 160 / 90$ & $\geq 160 / 90$ & $\geq 140 / 90$ & Thiazides & A \\
\hline WHO & $\geq 150 / 95$ & $\geq 140 / 90$ & $\geq 140 / 90$ & $\geq 130 / 85$ & $\begin{array}{l}\text { Low dose } \\
\text { monotherapy }\end{array}$ & \\
\hline VHA & $>160$ and $/$ or $>100$ & $>160$ and $/$ or $>100$ & $\geq 130 / 85$ & $\geq 130 / 85$ & $\begin{array}{l}\text { Thiazides or } \beta \text { - } \\
\text { blockers }\end{array}$ & \\
\hline SIGN & $\geq 160 / 100$ & $\geq 160 / 100$ & $\geq 140 / 90$ & $\geq 140 / 90$ & Thiazides & $A$ \\
\hline $\mathrm{ESH}$ & $\geq 150 / 95$ & $\geq 140 / 90$ & $\geq 130 / 85$ & $\geq 130 / 85$ & $\begin{array}{l}\text { Low dose } \\
\text { monotherapy or a } \\
\text { combination }\end{array}$ & \\
\hline ICSI & $\geq 160 / 100$ & $\geq 140 / 90$ & $\geq 130 / 85$ & $\geq 130 / 85$ & Thiazides & \\
\hline JNC & $\geq 140 / 90$ & $\geq 140 / 90$ & $\geq 130 / 80$ & $\geq 130 / 80$ & $\begin{array}{l}\text { Thiazides alone } \\
\text { and/or } \\
\text { combination ACE, } \\
\text { ARB, } \beta \text {-blockers, } \\
\text { CCB }\end{array}$ & \\
\hline SA & $\geq 140 / 90$ & $\geq 140 / 90$ & $\geq 130 / 85$ & $\geq 130 / 85$ & Thiazides & \\
\hline $\mathrm{BHS}$ & $\geq 160 / 100$ & $\geq 140 / 90$ & $\geq 140 / 90$ & $\geq 140 / 90$ & $\begin{array}{l}\text { Thiazides or } \\
\text { calcium channel } \\
\text { blockers }\end{array}$ & C \\
\hline NICE & $\geq 160 / 100$ & $\begin{array}{l}\geq 160 / 100 \text { or } \geq \\
140 / 90 \text { if } C H D \S \\
\text { risk } \geq 15 \% \text { or } \\
\text { CVDIrisk } \geq 20 \%\end{array}$ & $\geq 140 / 90$ & $\begin{array}{l}\text { Outside scope of } \\
\text { guideline }\end{array}$ & Thiazides & $A[34]$ \\
\hline
\end{tabular}

\footnotetext{
* Blood pressure threshold in $\mathrm{mmHg}$

† Thiazides: low dose thiazide diuretics

ACE: angiotensin-converting enzyme inhibitors

ARB: angiotensin receptor blockers

CCB: calcium channel blocker

\$CVD: cardiovascular disease

ICHD: coronary heart disease
}

BMI of $18.5-25 \mathrm{~kg} / \mathrm{m}^{2}$, restriction of salt intake to under $6 \mathrm{~g} /$ day and restriction of alcohol to 14 to $20 \mathrm{~g}$ (ethanol) per day for women and from 24 to $30 \mathrm{~g}$ (ethanol) per day for men. Differences in the daily limits for alcohol consumption may reflect the variations on guidance for sensible drinking in different countries. Most guidelines recommended a diet rich in fruit and vegetables with reduced saturated and total fats. Guidelines typically recommended 30-45 minutes of aerobic exercise three to five times per week. Although their recommendations were similar, guidelines lacked consistency in the estimations of the effect of lifestyle changes on blood pressure, possibly reflecting the different data sources used. Guidelines varied in the additional areas that they addressed: potassium, magnesium and calcium supplementation, management of stress and caffeine consumption were considered by some of the guidelines. This demonstrates one of the challenges facing guideline developers. Each clinical care pathway involving assessment, diagnosis, treatment and follow-up requires multiple complex deci- sions. A clinical guideline will be unable to offer guidance on every consideration that must be made by caregivers and patients. Guidelines will reflect this complexity and are likely to vary in their scope and coverage of the decisions involved in the care pathway.

\section{Criteria for initiation of antihypertensive drug therapy}

Guidelines used a varying combination of blood pressure and other factors to establish a threshold for drug therapy (see Table 5). These factors included the presence of concomitant disease, target organ damage, cardiovascular risk factors, response to lifestyle changes and the patient's own personal preferences. For uncomplicated patients the relatively recent SA and JNC guidelines recommended lower thresholds. All guidelines (except CMA) recommended lower thresholds for patients with target organ damage, renal disease or diabetes. Some guidelines modified their recommendations for older age groups and specific ethnic groups. 
Table 6: Reporting in guidelines of trials and systematic reviews of salt reduction

\begin{tabular}{|c|c|c|c|c|c|c|c|c|c|c|c|}
\hline $\begin{array}{l}\text { Referen } \\
\text { ce }\end{array}$ & Year & $\begin{array}{l}\text { CMA } \\
1999\end{array}$ & $\begin{array}{c}\text { WHO } \\
1999\end{array}$ & $\begin{array}{l}\text { VHA } \\
1999\end{array}$ & $\begin{array}{l}\text { SIGN } \\
2001\end{array}$ & $\begin{array}{l}\text { ESH } \\
2003\end{array}$ & $\begin{array}{l}\text { ICSI } \\
2003\end{array}$ & $\begin{array}{l}\text { JNC } \\
2003\end{array}$ & SA 2003 & $\begin{array}{l}\text { BHS } \\
2004\end{array}$ & $\begin{array}{l}\text { NICE } \\
2004\end{array}$ \\
\hline \multicolumn{12}{|c|}{ Randomised Controlled Trials } \\
\hline $\begin{array}{l}\text { Silman } \\
{[35]}\end{array}$ & 1983 & Y & & & & & & & & $\operatorname{SR}[36]$ & $Y$ \\
\hline $\begin{array}{l}\text { Fagerberg } \\
\text { [37] }\end{array}$ & 1985 & $\mathrm{SR}[38]$ & & & $\mathrm{SR}[38]$ & & & & & $\operatorname{SR}[38]$ & Y \\
\hline $\begin{array}{l}\text { Chalmers } \\
\text { [39] }\end{array}$ & 1986 & $\mathrm{SR}[38]$ & $\mathrm{SR}[40]$ & & $\mathrm{SR}[38]$ & & & & & $\operatorname{SR}[38]$ & Y \\
\hline $\begin{array}{l}\text { Chalmers } \\
{[41,42]}\end{array}$ & 1989 & & & & & $\operatorname{SR}[40]$ & & & & & Y \\
\hline TOHPI & 1992 & & & Y & & & & & & & $Y(e x)$ \\
\hline Jula [43] & 1994 & $\mathrm{SR}[38]$ & & & $\mathrm{SR}[38]$ & & & & & $\operatorname{SR}[38]$ & Y \\
\hline $\begin{array}{l}\text { Whelton } \\
\text { [44] }\end{array}$ & 1998 & & & & 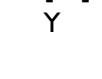 & & & & & $Y$ & $Y(e x)$ \\
\hline $\begin{array}{l}\text { Appel } \\
\text { [45] }\end{array}$ & 2001 & & & & & & $Y$ & & & & $Y(e x)$ \\
\hline $\begin{array}{l}\text { Sacks } \\
{[46]}\end{array}$ & 2001 & & & & & & & $Y$ & $Y$ & & $Y(e x)$ \\
\hline \multicolumn{12}{|c|}{ Systematic Reviews } \\
\hline Law [47] & 1991 & & & $Y$ & & & $Y$ & & & & $Y$ \\
\hline $\begin{array}{l}\text { Midgely } \\
\text { [38] }\end{array}$ & 1996 & Y & & & Y & & & & & Y & Y \\
\hline $\begin{array}{l}\text { Cutler } \\
{[40]}\end{array}$ & 1997 & & $Y$ & & & $\mathrm{Y}$ & & & & & $Y$ \\
\hline $\begin{array}{l}\text { Hooper } \\
{[36]}\end{array}$ & 2002 & & & & & & & & & $\mathrm{Y}$ & $Y$ \\
\hline \multicolumn{12}{|c|}{ Grade of recommendation } \\
\hline & & B & & I & $A$ & - & - & - & - & $A$ & $\mathrm{~B}[34]$ \\
\hline
\end{tabular}

Y Trial cited by guideline

SR Trial included in systematic review cited by guideline

$Y(e x)$ Trial cited by guideline but excluded from meta-analysis

First line drug therapies

Variations existed in thresholds for initiating drug treatment and initial drug therapy in typical patients (table 5). Five guidelines recommended the use of thiazdes as initial therapy in non- black patients aged over 55-65 years (SIGN, CMA, ICSI, SA, NICE). The other five differed in their recommendations, one recommended thiazides or b-blockers (VHA), one recommended low dose monotherapy selecting from a broad range of antihypertensive agents (WHO), one recommended low dose monotherapy or a combination of low dose antihypertensive agents (ESH), one recommended thiazides or calcium channel blockers (BHS) and one recommended thiazides alone or in combination with a range of antihypertensive drugs (JNC). The pattern of variation in these recommendations did not follow publication date of the guideline or relate to the research sources used in the development of the guideline.

\section{Grading recommendations and links to the evidence base} Salt intake

Although all guidelines recommended restriction of salt intake, four (SIGN, CMA, BHS, NICE) relied upon a similar and extensive body of work, either directly using the original data in a systematic review or indirectly sourcing the study via a previously published systematic review (see table 6). Nevertheless, these four guidelines were inconsistent in their grading of the recommendation: two guidelines (CMA, NICE) graded it 'B' suggesting that the pattern of care was recommended with caution and based upon research evidence subject to bias, while two graded it as ' $\mathrm{A}$ ' (BHS, SIGN) indicating that the recommendation was based on strong research evidence not vulnerable to bias. Although the VHA guideline cited much less evidence than these four guidelines, it nevertheless graded salt restriction as ' $\mathrm{I}$ ', equivalent to 'A' in other schemes. These disparities reflects the differences in the grading of recommendations in guideline development. Both the CMA and NICE guidelines adopted systems that required judgement about the quality of the RCT and the strength of its findings rather than a system that graded recommendations solely upon research design. The other five guidelines made similar recommendations about salt restriction, although they cited very limited evidence to support this. 
Table 7: Reporting in guidelines of trials of antihypertensive medication a

\begin{tabular}{|c|c|c|c|c|c|c|c|c|c|c|c|}
\hline Triala $^{a}$ & Yearb & CMA 1999 & $\begin{array}{l}\text { WHO } \\
1999\end{array}$ & $\begin{array}{l}\text { VHA } \\
1999\end{array}$ & $\begin{array}{l}\text { SIGN } \\
2001\end{array}$ & $\begin{array}{l}\text { ESH } \\
2003\end{array}$ & $\begin{array}{l}\text { ICSI } \\
2003\end{array}$ & $\begin{array}{l}\text { JNC } \\
2003\end{array}$ & SA 2003 & $\begin{array}{l}\text { BHS } \\
2004\end{array}$ & $\begin{array}{l}\text { NICE } \\
2004\end{array}$ \\
\hline VA-II[48] & 1970 & $\operatorname{SR}[49,50]$ & $\operatorname{SR}[49,51]$ & & $\begin{array}{l}\text { SR[49,5I, } \\
52]\end{array}$ & $\mathrm{SR}[5 \mathrm{I}]$ & & SR[53] & $\operatorname{SR}[5 \mathrm{I}]$ & SR[49] & $\checkmark$ \\
\hline $\begin{array}{l}\text { HSCSG[5 } \\
4]\end{array}$ & 1974 & $\mathrm{SR}[50]$ & $S R[5 I]$ & & $\mathrm{SR}[5 \mathrm{I}]$ & $S R[5 I]$ & & SR[53] & $\operatorname{SR}[51]$ & & $\checkmark$ \\
\hline $\begin{array}{l}\text { USPHS[55 } \\
\text { ] }\end{array}$ & 1977 & $\mathrm{SR}[50]$ & $\mathrm{SR}[5 \mathrm{I}]$ & & $\mathrm{SR}[5 \mathrm{I}]$ & $\mathrm{SR}[5 \mathrm{I}]$ & & $\mathrm{SR}[53]$ & $\operatorname{SR}[5 \mathrm{I}]$ & & $\checkmark$ \\
\hline $\begin{array}{l}\text { VA- } \\
\text { NHLBI[56 }\end{array}$ & 1978 & $\mathrm{SR}[50]$ & $\mathrm{SR}[5 \mathrm{I}]$ & & $\mathrm{SR}[5 \mathrm{I}]$ & $\mathrm{SR}[5 \mathrm{I}]$ & & SR[53] & $\operatorname{SR}[5 \mathrm{I}]$ & & $\checkmark$ \\
\hline $\begin{array}{l}\text { HDFP[57- } \\
59]\end{array}$ & 1979 & $\mathrm{SR}[49]$ & $\operatorname{SR}[49,51]$ & & $\begin{array}{l}\text { SR[49,5I, } \\
52,60]\end{array}$ & $\operatorname{SR}[5 I, 6 I]$ & $\checkmark$ & $\checkmark$ & $\operatorname{SR}[51]$ & $S R[49]$ & $\checkmark$ \\
\hline $\begin{array}{l}\text { ANBPS[6 } \\
\text { 2] }\end{array}$ & 1980 & & $S R[5 I]$ & & $\begin{array}{l}\mathrm{SR}[51,52, \\
60]\end{array}$ & $S R[51,63]$ & & SR & SR[5I] & & $\checkmark$ \\
\hline $\begin{array}{l}\text { EWPHE[6 } \\
4,65]\end{array}$ & 1985 & $\operatorname{SR}[49,50]$ & $\begin{array}{l}\text { SR[49,5I, } \\
66]\end{array}$ & & $\checkmark$ & $\operatorname{SR}[5 I, 61]$ & & $\mathrm{SR}[53]$ & $\operatorname{SR}[51]$ & $S R[49]$ & $\checkmark$ \\
\hline $\begin{array}{l}\text { IPPPSH[6 } \\
\text { 7] }\end{array}$ & 1985 & & $\checkmark$ & & & $\checkmark$ & & & & & $\checkmark$ \\
\hline$M R C[68]$ & 1985 & $S R[50]$ & $\checkmark$ & & $\mathrm{SR}[5 \mathrm{I}]$ & $\checkmark$ & & $\mathrm{SR}[53]$ & $\operatorname{SR}[51]$ & & $\checkmark$ \\
\hline $\begin{array}{l}\text { Coope[69 } \\
\text { ] }\end{array}$ & 1986 & $\mathrm{SR}[49]$ & $\begin{array}{l}\text { SR[49,5I, } \\
66]\end{array}$ & & $\checkmark$ & $\operatorname{SR}[5 \mathrm{I}, 6 \mathrm{I}]$ & & $\operatorname{SR}[53]$ & $\operatorname{SR}[51]$ & $\mathrm{SR}[49]$ & $\checkmark$ \\
\hline $\begin{array}{l}\text { OSLO }[70 \\
]\end{array}$ & 1986 & $\mathrm{SR}[50]$ & $S R[5 I]$ & & $\operatorname{SR}[5 \mathrm{I}]$ & $\mathrm{SR}[5 \mathrm{I}]$ & & $\operatorname{SR}[53]$ & $\operatorname{SR}[51]$ & & $\checkmark$ \\
\hline $\begin{array}{l}\text { SHEP- } \\
\text { P[7I-73] }\end{array}$ & 1986 & $\mathrm{SR}[50]$ & & & $S R[60]$ & $S R[6 I]$ & & SR[53] & & & $\checkmark$ \\
\hline $\begin{array}{l}\text { HAPPHY[ } \\
74]\end{array}$ & 1987 & $S R[50]$ & $\checkmark$ & & & & & & & & $\checkmark$ \\
\hline $\begin{array}{l}\text { MAPHY[7 } \\
5]\end{array}$ & 1988 & & & & & & & & & & $\checkmark$ \\
\hline $\begin{array}{l}\text { CAPPP[7 } \\
6,77]\end{array}$ & 1990 & & $\checkmark$ & & $\checkmark$ & $\checkmark$ & & & $\checkmark$ & $\checkmark$ & $\checkmark$ \\
\hline $\begin{array}{l}\text { SHEP[78- } \\
81]\end{array}$ & 1991 & $\checkmark$ & $\checkmark$ & & $\checkmark$ & & $\checkmark$ & $\checkmark$ & & $\checkmark$ & $\checkmark$ \\
\hline $\begin{array}{l}\text { STOP- } \\
\text { H[82] }\end{array}$ & 1991 & $\mathrm{SR}[49]$ & $\checkmark$ & & $\checkmark$ & $S R[6 I]$ & $\checkmark$ & $\mathrm{SR}[53]$ & & $\mathrm{SR}[49]$ & $\checkmark$ \\
\hline $\begin{array}{l}\text { SYST- } \\
\text { EUR[83- } \\
86]\end{array}$ & 1991 & $\checkmark$ & $\checkmark$ & & $\checkmark$ & SR [87] & $\checkmark$ & & & $\checkmark$ & $\checkmark$ \\
\hline $\begin{array}{l}\text { MRC- } \\
\text { O[88] }\end{array}$ & 1992 & $S R[50]$ & $\checkmark$ & & $\checkmark$ & $S R[6 I]$ & $\checkmark$ & SR[53] & & $S R[49]$ & $\checkmark$ \\
\hline $\begin{array}{l}\text { STOP- } \\
\mathrm{H} 2[89-91]\end{array}$ & 1993 & $\mathrm{SR}[92]$ & & & $\checkmark$ & $\begin{array}{l}\text { SR[63,87, } \\
93]\end{array}$ & $\checkmark$ & & & $\checkmark$ & $\checkmark$ \\
\hline $\begin{array}{l}\text { NICS- } \\
\text { EH[94,95] }\end{array}$ & 1994 & $\mathrm{SR}[92]$ & & & & $\begin{array}{l}\text { SR[63,87, } \\
93]\end{array}$ & & & & $\operatorname{SR}[63,96]$ & $\checkmark$ \\
\hline НОТ[97] & 1995 & & & & & & & & & & \\
\hline PATS[98] & 1995 & $\checkmark$ & $\checkmark$ & $\checkmark$ & $\checkmark$ & $\checkmark$ & $\checkmark$ & $\checkmark$ & & $\checkmark$ & $\checkmark$ \\
\hline $\begin{array}{l}\text { ALLHAT[ } \\
99,100]\end{array}$ & 1996 & & & & & $\checkmark$ & $\checkmark$ & $\begin{array}{l}\checkmark \\
\checkmark\end{array}$ & & $\checkmark$ & $\checkmark$ \\
\hline $\begin{array}{l}\text { MIDAS[I } \\
01,102]\end{array}$ & 1996 & SR $[50,92]$ & & & & $\checkmark$ & $\checkmark$ & & & $S R[63]$ & $\checkmark$ \\
\hline $\begin{array}{l}\text { VHAS[10 } \\
3]\end{array}$ & 1997 & $\operatorname{SR}[50,92]$ & $\checkmark$ & & & $\begin{array}{l}\text { SR }[3,87,9 \\
3]\end{array}$ & & & & $\operatorname{SR}[63,96]$ & $\checkmark$ \\
\hline
\end{tabular}


Table 7: Reporting in guidelines of trials of antihypertensive medication a (Continued)

\begin{tabular}{|c|c|c|c|c|c|c|c|c|c|c|}
\hline $\begin{array}{l}\mathrm{ABCD}[10 \\
4]\end{array}$ & 1998 & SR[92] & & & SR[87] & $\checkmark$ & $\checkmark$ & & $\checkmark$ & $\checkmark$ \\
\hline $\begin{array}{l}\text { FACET[I } \\
05]\end{array}$ & 1998 & $\mathrm{SR}[92]$ & & & & & & & & $\checkmark$ \\
\hline $\begin{array}{l}\text { UKPDS[I } \\
06]\end{array}$ & 1998 & & $\checkmark$ & $\checkmark$ & $\checkmark$ & $\checkmark$ & $\checkmark$ & $\checkmark$ & $\checkmark$ & $\checkmark$ \\
\hline HOPE[I0 & 2000 & 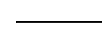 & & & & & & & & \\
\hline $\begin{array}{l}\text { INSIGHT[ } \\
\text { I08,109] }\end{array}$ & 2000 & $S R[92]$ & & 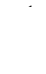 & $\checkmark$ & 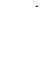 & & & $\checkmark$ & $\checkmark$ \\
\hline $\begin{array}{l}\text { NORDIL[ } \\
\text { II0] }\end{array}$ & 2000 & $S R[92]$ & & & $\checkmark$ & & & & $\checkmark$ & $\checkmark$ \\
\hline $\begin{array}{l}\text { IDNT[III } \\
\text { ] }\end{array}$ & 2001 & & & & SR[93] & & & & $\operatorname{SR}[96]$ & $\checkmark$ \\
\hline $\begin{array}{l}\text { PROGRE } \\
\text { SS[II2] }\end{array}$ & 2001 & & $\checkmark_{\mathrm{c}}$ & $\checkmark$ & $\checkmark$ & & $\checkmark$ & $\checkmark$ & $\checkmark$ & $\checkmark$ \\
\hline $\begin{array}{l}\text { RENAAL[ } \\
113]\end{array}$ & 2001 & & & & & & & & $\checkmark$ & $\checkmark$ \\
\hline ELSA[II4 & 2002 & & & - & SR[93] & & & & SR[96] & \\
\hline LIFE[II5] & 2002 & & & & & & & & & \\
\hline & & & & & $\checkmark$ & & $\checkmark$ & & $\checkmark$ & $\checkmark$ \\
\hline $\begin{array}{l}\text { ANBP2[1 } \\
16,117]\end{array}$ & 2003 & & & & SR[93] & & $\checkmark$ & & SR[96] & $\checkmark$ \\
\hline $\begin{array}{l}\text { CONVIN } \\
\text { CE[II8,I } \\
19]\end{array}$ & 2003 & & & & $\checkmark$ & & $\checkmark$ & & SR[96] & $\checkmark$ \\
\hline $\begin{array}{l}\text { SCOPE[I } \\
20]\end{array}$ & 2003 & & & & $\checkmark$ & & & & SR[96] & $\checkmark$ \\
\hline JMIC- & 2004 & & & & 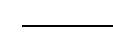 & & & & SR[96] & \\
\hline $\begin{array}{l}\text { VALUE[I } \\
22-124]\end{array}$ & 2004 & & & & & & & & & $\checkmark$ \\
\hline
\end{tabular}

a Trials post-dating the guidelines (indicated by the stepped line) were not available to the guideline developers.

b Year of earliest publication.

c Referred only to rationale and design.

$\checkmark$ Primary report of trial cited by guideline.

SR Trial included in systematic review cited by guideline; primary report not cited by guideline.

First line drug therapy

Overall the guidelines were relatively consistent in the studies that they cited as the evidence for the drug treatment recommendations (Table 7). One guideline (VHA) cited very little evidence; another (ICSI) did not cite any systematic reviews but referred to recent primary reports of trials, two guidelines (CMA, SA) relied almost exclusively on existing systematic reviews, whereas others (WHO, SIGN, ESH, JNC, BHS) supplemented citation of systematic reviews with citation of recent primary reports; one guideline (NICE) performed its own systematic review. Recommendations for use of thiazides and/or beta-blockers as initial drug therapy in typical patients were graded as 'A' by three guidelines (NICE, SIGN and $\mathrm{CMA}$ ). One guideline (BHS) recommended thiazides or calcium channel blockers grading it ' $\mathrm{C}$ '. This recommendation was largely based on the ABCD algorithm which in turn is based upon an extrapolation of how different drugs work rather than RCT findings; hence the evidence was graded as category III (descriptive studies, or evidence extrapolated from RCTs or quasi experimental studies), leading to the grade of ' $\mathrm{C}$ ' for the recommendation.

\section{Discussion}

Current guidelines are inconsistent in their handling of key methodologies that relate to the sourcing, interpretation and application of research evidence. Some cite a substantial body of evidence whereas others present little evidence. Some grade their recommendations - although the grading systems and grades used are not consistent whereas others do not. These findings are consistent with other studies exploring the quality of guideline development [24-27]. Methodological failings may affect the quality of the guideline in several ways. A search that is insufficiently thorough may introduce bias into the summary of the evidence [28]. Systematic reviews have been described as the optimum method of summarising evidence of effectiveness within a clinical practice guideline [29]. In this study we found that most of the guidelines relied on previously published systematic reviews, despite 
the possible problems with this strategy. Firstly, systematic reviews may date quickly and not incorporate newer evidence. Secondly, the scope of the published reviews may not always match the remit of the guideline and so may not be relevant to the target population of the guideline. Thirdly, up-to-date high quality systematic reviews may not be available in all the areas covered by a guideline.

Despite the inconsistent approach in the guidelines to sourcing the evidence, interpreting it and applying it to recommendations, and the great variation in the volume of supporting evidence cited, the areas of consensus are substantial. Different hypertension guidelines made similar recommendations for many areas of management, notably recommendations for lifestyle changes and their role as first line interventions for patients in certain categories of risk. This level of agreement suggests the possibility either that the published guideline did not cite all the evidence which influenced the recommendations, or that guideline groups may develop guidelines that are heavily influenced by previously published guidelines or an implicit international consensus. Guidelines generated without a systematic search of the literature and without systematic review of all the supporting evidence would be more likely to reflect the biases of developers and it would not be surprising if they were congruent with other guidelines in the same area.

Only five guidelines graded the recommendations made. Failure to grade research evidence and the subsequent recommendations means that the decision making process is not explicit and does not inform the guideline user of the strength of evidence underpinning a particular pattern of care.

The inconsistent grading of the same recommendation in different guidelines also indicates varying approaches to interpreting and applying research evidence in guideline development. The process of evaluating the quality of research evidence and applying this to guideline recommendations using a system of grading is clearly inconsistent and currently an evolving area of guideline methodology.

As well as seeking research evidence, guidelines seek to elicit and incorporate the views of clinical experts and various stakeholders in interpreting the evidence or in offering expert opinion where objective evidence is sparse. Indeed this is an important feature used to assess the quality of a guideline [30]. Differences in guidelines, reflecting the differing views of individuals participating in the guideline development process, are therefore to be expected [29,31]. Herein lies a tension between the rigour needed to try and produce objective and unbiased state- ments and to also be responsive to the views of participants. It is clear from other reviews of clinical guidelines that the composition of the development group is reflected in the recommendations. Savoie et al [31] in their critical appraisal of guidelines for cholesterol testing found that the greater the involvement of clinical experts in the development process of the guideline, the less the recommendations reflected the research evidence. As only three of the guidelines which we considered fully reported the composition of the guideline development group, it is not possible to make inferences about its impact on recommendations for hypertension. Achieving evidence based guidelines while incorporating the views of the various stakeholders within the development group may create conflict and divergence that the final guideline may mask. The differences between the guidelines described here may reflect this tension.

Differences in recommendations may reflect not only differences in material sourced, differences in interpreting and grading the evidence but also different influences in moving from evidence to recommendations. Differing recommendations for first line drug therapy suggest this. The research base underpinning the recommendations for first line drug therapies is strong in terms of the number and quality of trials in the area (see table 6). Nevertheless, three guidelines (NICE, SIGN and CMA) recommended thiazides for the uncomplicated patient, grading this as ' $\mathrm{A}$ ', while one (BHS) recommended thiazides or calcium channel blockers grading it ' $\mathrm{C}$ '. This suggests either a strong competing interest or the possibility that a less supported but broader recommendation is felt to hold greater clinical merit than older treatments which have accumulated a strong research base. This again may reflect a tension in the development of guidelines, between the restrictiveness of the conventional evidence based approach which inevitably relies on older, well researched therapies and the greater openness of an approach which allows newer, less well endorsed treatments.

Clinical guidelines are rarely based solely on the research evidence and incorporate the consensus views of experts. Raine et al. argue that current approaches to guideline development often lack a sufficient transparency and reliability concerning how such consensus opinions are formed [32]. They highlight the possible influence of key individuals, unrepresentative decision making and the role of constraints of time and resources which limit the range of guidelines that can be generated and their need for updating. They propose an approach which makes reasons for disagreement and degree of consensus explicit and suggests the inclusion of a survey stage to enhance reliability. 
The potential influence of external pressures in the formation of guideline recommendations highlights the need for transparency in the declarations of conflictions of interest by authors of clinical practice guidelines. One investigation of panels that write clinical guidelines found that more than one-third of authors declared financial links to relevant drug companies, with around $70 \%$ of panels being affected [33]. Another study found that $87 \%$ of authors of clinical practice guidelines had some form of interaction with the pharmaceutical industry [7]. If authors have relationships that pose a potential conflict of interest these need appropriate disclosure so that readers may evaluate the merit of those guidelines.

\section{Conclusion}

Many challenges exist to improve the use of evidence in all its forms in guideline development. Clinical practice guidelines remain a developing healthcare technology and if they are to fulfil their potential as a tool to improve standards of care these challenges need to be addressed. The requirements of future guidelines are clear if they are to inform clinicians and patients about appropriate healthcare. Authoritative and rigorously developed guidelines should (where possible) feature transparent and fully reported: guideline group methods and participation; involvement of stakeholders and sponsors; reporting and use of evidence and linking of recommendation to evidence; understanding of health care delivery, the policy context and narratives of patient experience.

\section{Abbreviations}

ACE angiotensin converting enzyme

ARB angiotensin receptor blocker

BMI body mass index

g gram

kg kilogram

m metre

mmHg millimetres of mercury

mins minutes

\section{Competing interests}

The authors contributed to the development of one of the guidelines reviewed [26].

\section{Authors' contributions}

FC, HOD and JMM wrote the manuscript.

ME critically revised the manuscript.
FC, JC, FRB, HOD and JMM performed data abstraction.

FC and FRB performed literature searches.

JMM designed the study.

\section{Acknowledgements}

This work was undertaken with funding from the National Institute for Clinical Excellence. However, the views expressed are those of the authors and not the funding body.

\section{References}

I. Field MJ, Lohr KN, (eds): Guidelines for clinical practice: from development to use Washington DC, National Academy Press; 1992.

2. Dans PE: Credibility, cookbook medicine, and common sense: guidelines and the college. Annals of Internal Medicine 1994, I 20:966-968.

3. Guidelines CCP: Clinical practice guidelines: directions for a new program Edited by: Field MG LKN. Washington, National Academy Press, Institute of Medicine; 1990.

4. Eccles M, Freemantle N, Mason J: Developing cost effectiveness guidelines. BM] 1997:27-27.

5. Collaboration TAGREE: Development and validation of an interational appraisal instrument for assessing the quality of clinical practice guidelines: the AGREE project. Quality and Safety in Health Care 2003, 12:18-23.

6. Burgers J, Grol R, Klazinga N, Van Der Bij A, Makela M, Zaat J, Ollenschlager G, Grilli R, Slutsky JPNC, Bruun Madsen P, Durieus P, Brouwers M, Jackson R, Meulenberg F, Ten Have P, Fervers B, Grimshaw J, Burnand B, Miller J, Makela J, Dosquet P, Eccles M, Silagy C, Bowersox J, Irwin P, Dahlgren H, Atkins D: International comparison of 19 clinical guideline programmes - a survey of the AGREE Collaboration. Zeitschrift fur Arzliche Fortbildung und Qualitatssicherung 2003, 97:8I-88.

7. Choudhry NK, Stelfox HT, Detsky AS: Relationships between authors of clinical practice guidelines and the pharmaceutical industry. JAMA 2002, 287:6I2-617.

8. Editorial: Just how tainted has medicine become? The Lancet 2002, 359: I 167-II67.

9. WHO: The world health report 2002: reducing risks, promoting healthy life. Geneva; 2002.

10. Statistics ON: Key Health Statistics from General Practice 1998: analysis of morbidity and treatment data, including time trends, England and Wales London, National Statistics; 2000.

II. Collaboration PS: Age-specific relevance of usual blood pressure to vascular mortality: a meta-analysis of individual data for one million adults in $6 \mathrm{I}$ prospective studies. Lancet 2002, 360:1903-1913.

12. Primatesta P, Brookes M, Poulter NR: Improved hypertension management and control: results from the health survey for England 1998. Hypertension 200I, 38:827-832.

13. Task force for the Development of the 1999 Canadian recommendations for the management of hypertension: 1999 Canadian recommendations for the management of hypertension including case-based applications of the recommendations. Can Med Assoc J 1999, I6I:SI-S22.

14. WHO: 1999 World Health Organization-International Society of Hypertension Guidelines for the Management of Hypertension. Guidelines Subcommittee. J Hypertens 1999, I7:151-183.

15. Workgroup TH: VHA/DOD Clinical Practice Guideline for diagnosis and management of hypertension in the primary care setting. 1999 [http://www.va.gov/health/hypertension/ HTN.doc]. USA, Veteran's Health Administration and Department of Defense

16. Improvement ICS: Hypertension diagnosis and treatment. ICSI; 2003:I-46.

17. Southern Africa Hypertension Society Hypertension Guideline Working Groups 2000 and 2003: South African Hypertension Guideline 2003 update. South Africa, Southern Africa Hypertension Society; 2003:i-32. 
18. Network SIG: Hypertension in older people. Edinburgh, SIGN; 200I:I-50.

19. Committee G: 2003 European Society of Hypertension-European Society of Cardiology guidelines for the management of arterial hypertension. J Hypertens 2003, 21:1011-1053.

20. Chobanian AV, Bakris GL, Black HR, Cushman WC, Green LA, Izzo JL, Jones DW, Materson BJ, Oparil S, Wright JTJ, Roccella EJ, National Heart LBIJNCPDETHBP, National High Blood Pressure Education Program Coordinating Committee.: The Seventh Report of the Joint National Committee on Prevention, Detection, Evaluation, and Treatment of High Blood Pressure: the JNC 7 report. JAMA 2003, 289:2560-2572.

21. Williams B, Poulter N, Brown MJ, Davis M, Mclnnes GT, Poulter N, Potter JP: The BHS Guidelines Working Party Guidelines for Management of Hypertension: Report of the Fourth Working Party of the British Hypertension Society, 2004 - BHS IV. J Hum Hypertens 2004, 18:139-185.

22. North of England Hypertension Guideline Development Group: Essential hypertension: managing adult patients in primary care. Newcastle, Centre for Health Services Research, University of Newcastle; 2004.

23. Atkins D, Eccles M, Flottorp S, Guyatt GH, Henry D, Hill S, Liberati A, O'Connell D, Oxman AD, Phillips B, Schunemann H, Tan-Torres Edejer T, Vist GE, Williams Jr JW, Group TGW: Systems for grading the quality of evidence and the strength of recommendations I: critical appraisal of existing approaches The Grade Working Group. BMC Health Services Research 2004, 4:

24. Grilli R, Magrini N, Penna A, Mura G, Liberati A: Practice guidelines developed by specialty societies: the need for a critical appraisal. Lancet 2000, 355:103-106.

25. Thomson R, McElroy H, Sudlow M: Guidelines on anticoagulant treatment in atrial fibrillation in Great Britain: variation in content and implications for treatment. BMJ 1998 316:509-513.

26. Fretheim A, Williams JWJ, Oxman AD, Herrin J: The relation between methods and recommendations in clinical practice guidelines for hypertension and hyperlipidemia. I Fam Pract 2002, 51:963-968.

27. McAlister FA, Zarnke KB, Campbell NR, Feldman RD, Levine M, Mahon J, Grover SA, Lewanczuk R, Leenen F, Tobe S, Lebel M, Stone J, Schiffrin EL, Rabkin SW, Ogilvie RI, Larochelle P, Jones C, Honos G, Fodor G, Burgess E, Hamet P, Herman R, Irvine J, Culleton B, Wright JM, Group. CHRW: The 200I Canadian recommendations for the management of hypertension: Part two--Therapy. Can J Cardiol 2002, 18:625-64I.

28. Dickersin K, Scherer R, Lefebvre C: Systematic reviews: identifying relevant studies for systematic reviews. BM] 1994 309: |286-|29|.

29. Eccles M, Freemantle N, Mason J: Using systematic reviews in clinical guideline development. In Systematic Reviews in Health Care Edited by: M E, G DS and DG A. BMJ publishing group; 200I:400-409.

30. Cluzeau F, Littlejohns P, Grimshaw J, Feder G: Appraisal Instrument for Clinical Guidelines. London, St George's Hospital Medical School; 1997.

31. Savoie I, Kazaniian A Bassett K. Do clinical practice guidelines reflect research evidence? J health Serv Res Policy 2000, 5:76-82.

32. Raine R, Sanderson C, Black N: Developing clinical guidelines: a challenge to current methods. British Medical Journal 2006, $331: 63 \mid-633$.

33. Taylor R, Giles J: Cash interests taint drug advice. Nature 2005 437: 1070-107|.

34. Mason J, Eccles M: Guideline recommendation an evidence grading (GREG): a new grading method for clinical guideline development groups. Volume Report 109. Newcastle upon Tyne, Centre for Health Services Research, University of Newcastle upon Tyne; 2003.

35. Silman AJ, Locke C, Mitchell P, Humpherson P: Evaluation of the effectiveness of a low sodium diet in the treatment of mild to moderate hypertension. Lancet 1983, I: I I79-1 I82.

36. Hooper L, Bartlett C, Davey Smith G, Ebrahim S: Systematic review of long term effects of advice to reduce dietary salt in adults. $B M]$ 2002, 325:628-636.

37. Fagerberg B, Andersson OK, Persson B, Hedner T: Reactivity to norepinehrine and effect of sodium on blood pressure during weight loss. Hypertension 1985, 7:586-592.
38. Midgley JP, Matthew AG, Greenwood CM, Logan AG: Effect of reduced dietary sodium on blood pressure: a meta-analysis of randomized controlled trials. JAMA 1996, 275:1590-1597.

39. Chalmers J, Morgan T, Doyle A, Dickson B, Hopper J, Mathews J, Matthews J, Moulds R, Myers J, Nowson C, Scoggins B, Stebbing M: Australian National Health and Medical Research Council dietary salt study in mild hypertension. I Hypertens 1986, 4:S629-S637.

40. Cutler JA, Follmann D, Allender PS: Randomized trials of sodium reduction: an overview. Am J Clin Nutr 1997, 65:643S-65IS.

41. Australian National Health and Medical Research Council Dietary Salt Study Management Committee: Effects of replacing sodium intake in subjects on a low sodium diet: a crossover study. Clin Exp Hypertens 1989, A I I: 101 I-1024.

42. Australian National Health and Medical Research Council Dietary Salt Study Management Committee: Fall in blood pressure with modest reduction in dietary salt intake in mild hypertension. Lancet 1989, I:399-402.

43. Jula AM, Karanko HM: Effects on left-ventricular hypertrophy of long-term nonpharmacolgical treatment with sodium restriction in mild-to-moderate essential hypertension. Circulation 1994, 89: 1023-1031.

44. Whelton PK, Appel LJ, Espeland MA, Applegate WB, Ettinger WH, Kostis JB, al : Sodium reduction and weight loss in the treatment of hypertension in older persons: a randomized controlled Trial of Nonpharmacologic Interventions in the Elderly (TONE). JAMA 1998, 279:839-846.

45. Appel LJ, Espeland MA, Easter L, Wilson AC, Folmar S, Lacy CR Effects of reduced sodium intake on hypertension control in older individuals: results from the Trial of Nonpharmacologic Interventions in the Elderly (TONE). Arch Intern Med 200I, 16 I:685-693.

46. Sacks FM, Svetkey LP, Vollmer WM, Appel LJ, Bray GA, Harsha D, Group DASHSCR: Effects on blood pressure of reduced dietary sodium and the Dietary Approaches to Stop Hypertension (DASH) diet. N Engl J Med 200I, 344:3-I0.

47. Law MR, Frost CD, Wald NJ: III Analysis of data from trials of salt reduction. $B M] 1991,302: 819-824$.

48. Veterans Administration Cooperative Study Group on Antihypertensive Agents: Effects of treatment on morbidity in hypertension: II results in patients with diastolic blood pressure averaging 90 through II 4 mm Hg. JAMA 1970, 2I 3: | |43-I I 52.

49. Messerli FH, Grossman E, Goldbourt U: Are beta-blockers efficacious as first-line therapy for hypertension in the elderly? $A$ systematic review. JAMA 1998, 279:1903-1907.

50. Wright JM, C. L, G.K C: Systematic review of antihypertensive therapies. Canadian Medical Associataion Journal 1999, I6I:

5I. Collins R, Peto R, MacMahon S, Herbert P, Fiebach NH, Eberlein KA Godwin J, Qizilbash N, Taylor JO, Hennekens $\mathrm{CH}$ : Blood pressure, stroke and coronary heart disease: Part 2, short-term reductions in blood pressure: overview of randomised drug trials in their epidemiology context. Lancet 1990, 335:827-838.

52. Insua JT, H.S. S, Lau T.Lau J.Reitman D.Pagano D.Chalmers T.C.: Drug Treatment of Hypertension in the Elderly. A MetaAnalysis. Ann Intern Med 1994, I 2 I:355-362.

53. Psaty BM, Smith NL, Siscovick DS, Koepsell TD, Weiss NS, Heckbert SR, Lemaitre RN, Wagner EH, Furberg CD: Health outcomes associated with antihypertensive therapies used as first-line agents: a systematic review and meta-analysis. JAMA 1997, 277:739-745

54. Group HSCS: Effect of antihypertensive treatment on stroke recurrence. JAMA 1974, 229:409-4I8.

55. Smith WM: Treatment of mild hypertension: results of a tenyear intervention trial. Circ Res 1977, 40:98-105.

56. Prepared for the Veterans Administration-National Heart LBISGETMH: Evaluation of drug treatment in mild hypertension: VA-NHLBI feasibility trial. Plan and preliminary results of a two-year feasibility trial for a multicenter intervention study to evaluate the benefits versus the disadvantages of treating mild hypertension. Ann N Y Acad Sci 1978, 304:267-288.

57. Hypertension Detection and Follow-up Program Cooperative Group: Five-year findings of the hypertension detection and followup program: I reduction in mortality of persons with high blood pressure, including mild hypertension. JAMA 1979, 242:2562-257। 
58. Hypertension Detection and Follow-up Program Cooperative Group: Five-year findings of the Hypertension Detection and Follow-up Program: III reduction in stroke incidence among persons with high blood pressure. JAMA 1982, 247:633-638.

59. Hypertension Detection and Follow-up Program Cooperative Group: Effect of stepped care treatment on the incidence of myocardial infarction and angina pectoris: 5-year findings of the Hypertension Detection and Follow-up Program. Hypertension 1984, 6:I-I98-I-206.

60. Pearce KA, C.D F, J R: Does Antihypertenive Treatment of the Elderly prevent Cardiovascular Events or Prolong Life?: A Meta-analysis of Hypertension Treatment Trials. Arch Fam Med 1995, 4:943-950.

6I. Gueyffier F, Boutitie F, Boissel JP, Pocock S, Coope J, Cutler J, Ekbom $\mathrm{T}$, al : Effect of antihypertensive drug treatment on cardiovascular outcomes in women and men: a meta-analysis of individual patient data from randomized controlled trials. Ann Intern Med 1997, I 26:76I-767.

62. Australian National Blood Pressure Management Committee:: The Australian Therapeutic Trial in Mild Hypertension. Lancet 1980, I:I26|-1267.

63. J.A S, J W, L T: Cardiovascular protection and blood pressure reduction: a meta-analysis. The Lancet 200I, 358:|305-I3I5.

64. European Working Party on High Blood Pressure in the Elderly (EWPHE): An international trial of antihypertensive therapy in elderly patients: objectives protocol and organization. Arch Int Pharmacodyn Ther 1985, 275:300-334.

65. Staessen J, Bulpitt C, Clement D, de Leeuw P, Fagard R, Fletcher A, Forette F, Leonetti G, Nissinen A, O'Malley K, Tuomilehto J, Webster J, Williams BO: Relation between mortality and treated blood pressure in elderly patients with hypertension: report of the European Working Party on High Blood Pressure in the Elderly. BMJ I989, 298:I552-I556.

66. MacMahon S, Rodgers A: The Effects of Blood Pressure Reduction in Older Patients: an overview of Five Randomized Controlled Trials in Elderly Hypertensives. Clin Exp Hypertens 1993, I 5:967-978.

67. Group IPPPSHC: Cardiovascular risk and risk factors in a randomized trial of treatment based on the beta-blocker oxprenolol: the International Prospective Primary Prevention Study in Hypertension (IPPPSH). J Hypertens 1985, 3:379-392.

68. Party MRCW: MRC trial of treatment of mild hypertension: principal results. BMJ 1985, 291:97-104.

69. Coope J, Warrender TS: Randomized trial of treatment of hypertension in elderly patients in primary care. BMJ 1986 , 293: II45-1152

70. Helgeland A: Treatment of mild hypertension: a five year controlled drug trial: The Oslo Study. Am J Med I 980, 69:725-732.

7I. Hulley SB, Furberg CD, Gurland B, McDonald R, Perry HM, Schnaper HW: Systolic Hypertension in the Elderly Program (SHEP) antihypertensive efficacy of chlorthalidone. Am J Cardiol 1985, 56:913-920.

72. Perry HM, McDonald RH, Hulley SB, Smith WM, Furberg CD, Greenlick MR: Systolic Hypertension in the Elderly Program. Pilot Study (SHEP-PS): morbidity and mortality experience. Hypertens 1986, 4:S2I-S23.

73. Perry HM, Smith WM, McDonald RH, Black D, Cutler JA, Furberg $C D$ : Morbidity and mortality in the Systolic Hypertension in the Elderly Program (SHEP) Pilot Study. Stroke 1989, 20:4-I3.

74. Wilhelmsen L, Berglund G, Elmfeldt D, Fitzsimons T, Holzgreve H, Hosie J, Hörnkvist PE, Pennert K, Tuomilehto J, Wedel H: Beta Blockers versus diuretics in hypertensive men: main results from the HAPPHY Trial. J Hypertens 1987, 5:56I-572.

75. Wikstrand J, Warnold I, Olsson G, Tuomilehto J, Elmfeldt D, Berglund G: Primary prevention with Metoprolol in patients with hypertension: mortality results from the MAPHY study. JAMA 1988, 259:1976-1982.

76. Hansson L, Lindholm LH, Niskanen L, Lanke J, Hedner T, Niklason A, Luomanaki K, Dahlöf B, de Faire U, Morlin C, Karlberg BE, Wester PO, Bjorck JE: Effect of angiotensin-converting-enzyme inhibition compared with conventional therapy on cardiovascular morbidity and mortality in hypertension: the Captopril Prevention Project (CAPPP) randomised trial. Lancet 1999 353:6|1-616.

77. group TCAPPP: The Captopril Prevention Project: a prospective intervention trial of angiotensin converting enzyme inhi- bition in the treatment of hypertension. J Hypertens 1990 , 8:985-990.

78. Perry HM, Davis BR, Price TR, Applegate WB, Fields WS, Guralnik JM, Kuller L, Pressel S, Stamler J, Probsfieldt JL: Effect of treating isolated systolic hypertension on the risk of developing various types and subtypes of stroke: the Systolic Hypertension in the Elderly Program (SHEP). JAMA 2000, 284:465-47I.

79. Group SHEPCR: Rationale and design of a randomized clinical trial on prevention of stroke in isolated systolic hypertension. I Clin Epidemiol 1988, 41:1 197-1208.

80. Group SHEPCR: Prevention of stroke by antihypertensive drug treatment in older persons with isolated systolic hypertension: final results of the Systolic Hypertension in the Elderly Program (SHEP). JAMA I99I, 265:3255-3264.

8I. Vaccarino V, Berger AK, Abramson J, Black HR, Setaro JF, Davey JA, Krumholz HM: Pulse pressure and risk of cardiovascular events in the systolic hypertension in the elderly program. Am / Cardiol 200I, 88:980-986.

82. Dahlöf B, Lindholm LH, Hansson L, Scherstén B, Ekbom T, Wester P: Morbidity and mortality in the Swedish Trial in Old Patients With Hypertension (STOP-Hypertension). Lancet 1991, 338: $1281-1285$.

83. Amery A, Birkenhager W, Bulpitt C], Clement D, de Leeuw P, Dollery CT: Syst-Eur: a multicenter trial on the treatment of isolated systolic hypertension in the elderly objectives, protocol, and organisation. Ageing 1991, 3:287-302.

84. Staessen JA, Fagard R, Thijs L, Celis H, Arabidze GG, Birkenhager WH, Bulpitt C], de Leeuw P, Dollery CT, Fletcher AE, Forette F, Leonetti G, Nachev C, O'Brien ET, Rosenfeld J, Rodicio JL, Tuomilehto J, Zanchetti A: Randomised double-blind comparison of placebo and active treatment for older patients with isolated systolic hypertension. Lancet 1997, 350:757-764.

85. Bulpitt CJ, Beckett NS, Fletcher AE, Thijs L, Staessen JA, Dumitrascu DL, Forette F, Leonetti G, Nachev C, Tuomilehto J, Fagard RH, investigators TSE: Withdrawal from treatment in the Syst-Eur Trial. J Hypertens 2002, 20:339-346.

86. Staessen JA, Thijs L, Birkenhager WH, Bulpitt CJ, Fagard R: Update on the Systolic Hypertension in Europe (Syst-Eur) Trial. Hypertension 1999, 33:1476-1477.

87. Blood Pressure Lowering Treatment Trialists' Collaboration: Effects of different blood-pressure-lowering regimens on major cardiovascular events: Results of prospectively-designed overviews of randomised trials. Lancet 2003, 362:1527-1535.

88. Party MRCW: Medical Research Council Trial of treatment of hypertension in older adults: principal results. BMJ 1992 , 304:405-4I2.

89. Dahlöf B, Hansson L, Lindholm LH, Schersten B, Wester PO, Ekbom T, Hedner T, de Faire U: STOP-Hypertension-2: a prospective intervention trial of newer versus older treatment alternatives in old patients with hypertension. Blood Press 1993. 2:|36-|4|.

90. Hansson L, Lindholm LH, Ekbom T, Dahlöf B, Lanke J, Schersten B, Wester PO, Hedner T: Randomised trial of old and new antihypertensive drugs in elderly patients: cardiovascular mortality and morbidity the Swedish Trial in Old Patients with Hypertension-2 study. Lancet 1999, 354:175|-I756.

91. Hansson L: Results of the STOP-Hypertension-2 trial. Blood Press 2000, 9:17-20

92. Pahor M, Psaty BM, Alderman MH, Applegate WB, Williamson JD, Cavazzini C, Furberg CD: Health outcomes associated with calcium antagonists compared with other first-line antihypertensive therapies: a meta-analysis of randomised controlled trials. Lancet 2000, 356:1949-1954.

93. Staessen JA, Wang JG, Thijs L: Cardiovascular prevention and blood pressure reduction: A quantitative overview updated until I March 2003. J Hypertens 2003, 2 I: I055-1076.

94. Kuwajima I, Kuramoto K, Ogihara T, limura O, Abe K, Saruta T, al : Tolerability and safety of a calcium channel blocker in comparison with a diuretic in the treatment of elderly patients with hypertension: Secondary analysis of the NICS-EH. Hypertens Res 200I, 24:475-480.

95. National Intervention Cooperative Study in Elderly Hypertensives Study Group: Randomized double-blind comparison of a calcium antagonist and a diuretic in elderly hypertensives. Hypertension 1999, 34: I I29-I I33. 
96. Blood Pressure Lowering Treatment Trialists' Collaboration: Effects of ACE inhibitors, calcium antagonists, and other bloodpressure-lowering drugs: results of prospectively designed overviews of randomised trials. Lancet 2000, 356:1955-1964.

97. Hansson L, Zanchetti A, Carruthers SG, Dahlöf B, Elmfeldt D, Julius $\mathrm{S}$, Menard J, Rahn KH, Wedel H, Westerling S: Effects of intensive blood-pressure lowering and low-dose aspirin in patients with hypertension: principal results of the Hypertension Optimal Treatment (HOT) randomised trial. Lancet 1998, 351:1755-1762

98. Group PATSC: Post-stroke antihypertensive treatment study: a preliminary result. Chin Med J (Engl) 1995, I 08:7I 0-7I7.

99. Davis BR, Cutler JA, Gordon DJ, Furberg CD, Wright JTJ, Cushman WC, Grimm RH, LaRosa J, Whelton PK, Perry HM, Alderman MH, Ford CE, Oparil S, Francis C, Proschan M, Pressel S, Hawkins CM Rationale and design for the Antihypertensive and Lipid Lowering Treatment to Prevent Heart Attack Trial (ALLHAT). Am J Hypertens 1996, 9:342-360.

100. The Antihypertensive and Lipid-Lowering Treatment to Prevent Heart Attack Trial (ALLHAT): Major outcomes in high-risk hypertensive patients randomized to angiotensin-converting enzyme inhibitor or calcium channel blocker vs diuretic. JAMA - Express 2002, 288:298I-2997.

101. Borhani NO, Mercuri M, Borhani PA, Buckalew VM, Canossa-Terris M, Carr AA, Kappagoda MD, Rocco MV, Schnaper HW, Sowers JR, Bond MG: Final outcome results of the Multicenter Isradipine Diuretic Atherosclerosis Study (MIDAS). JAMA 1996, 276:785-79l.

102. Byington RP, Furberg CD, Craven TE, Pahor M, Sowers JR: Isradipine in prediabetic hypertensive subjects. Diabetes Care 1998, 2 I:2103-2 I 10.

103. Rosei EA, Dal Palú C, Leonetti G, Magnani B, Pessina A, Zanchetti A: Clinical results of the Verapamil in Hypertension and Atherosclerosis Study. J Hypertens 1997, I 5: I 337-I 344.

104. Estacio RO, Jeffers BW, Hiatt WR, Biggerstaff SL, Gifford N, Schrier RW: The effect of nisoldipine as compared with enalapril on cardiovascular outcomes in patients with non-insulindependent diabetes and hypertension. N Engl J Med 1998, 338:645-652.

105. Tatti P, Pahor M, Byington RP, Di M, Guarisco R, Strollo G: Outcome results of the Fosinopril versus Amlodipine Cardiovascular Events Randomized Trial (FACET) in patients with hypertension and NIDDM. Diabetes Care 1998, 2 I:597-603.

106. Group UKPDS: Efficacy of atenolol and captopril in reducing risk of macrovascular and microvascular complications in type 2 diabetes: UKPDS 39. BMJ I998, 3 I 7:7। 3-720.

107. The Heart Outcomes Prevention Evaluation Study Investigators: Effects of an angiotensin-converting-enzyme inhibitor, Ramipril, on cardiovascular events in high-risk patients. $N \mathrm{EnglJ}$ Med 2000, 342: | 45-I53.

108. Brown MJ, Palmer CR, Castaigne A, de Leeuw PW, Mancia G, Rosenthal $\mathrm{T}$ : Morbidity and mortality in patients randomised to double-blind treatment with a long-acting calcium-channel blocker or diuretic in the International Nifedipine GITS study: Intervention as a Goal in Hypertension Treatment (INSIGHT). Lancet 2000, 356:366-372.

109. Brown MJ, Palmer CR, Castaigne A, de Leeuw PW, Mancia G, Rosenthal T: Principal results from the International Nifedipine GITS Study: Intervention as a Goal in Hypertension Treatment (INSIGHT). Eur Heart J 200I, 3:B20-B26.

I 10. Hansson L, Hedner T, Lund-Johansen P, Kjeldsen SE, Lindholm LH Syvertsen JO, Lanke J, de Faire U, Dahlöf B, Karlberg BE: Randomised trial of effects of calcium antagonists compared with diuretics and beta-blockers on cardiovascular morbidity and mortality in hypertension: the Nordic Diltiazem (NORDIL) study. Lancet 2000, 356:359-365.

III. Berl T, Hunsicker LG, Lewis JB, Pfeffer MA, Porush JG, Rouleau JL, Drury PL, Esmatjes E, Hricik D, Parikh CR, Raz I, Vanhille P, Wiegmann TB, Wolfe BM, Locatelli F, Goldhaber SZ, Lewis EJ, Irbesartan Diabetic Nephropathy Trial.Collaborative Study Group.: Cardiovascular outcomes in the Irbesartan Diabetic Nephropathy Trial of patients with type 2 diabetes and overt nephropathy. Ann Intern Med 2003, I 38:542-549.

I 12. Group PROGRESSC: Randomised trial of a perindopril-based blood-pressure-lowering regimen among 6105 individuals with previous stroke or transient ischaemic attack. Lancet 200I, 358:1033-104I.

I 13. Brenner BM, Cooper ME, de Zeeuw D, Keane WF, Mitch WE, Parving H, Remuzzi G, Snapinn SM, Zhang Z, Shahinfar S: Effects of losartan on renal and cardiovascular outcomes in patients with type 2 diabetes and nephropathy. N Engl J Med 200I, 345:86I-869.

I14. Zanchetti A, Bond MG, Hennig M, Neiss A, Mancia G, Dal Palu C, Hansson L, Magnani B, Rahn KH, Reid JL, Rodicio J, Safar M, Eckes L, Rizzini P, European Lacidipine Study on Atherosclerosis investigators.: Calcium antagonist lacidipine slows down progression of asymptomatic carotid atherosclerosis: principal results of the European Lacidipine Study on Atherosclerosis (ELSA), a randomized, double-blind, long-term trial. Circulation 2002, I 06:2422-2427.

I I5. Dahlöf B, Devereux RB, Kjeldsen SE, Julius S, Beevers G, de Faire U, Fyhrquist $F$, Ibsen $H$, Kristiansson $K$, Lederballe-Pedersen $O$, Lindholm LH, Nieminen M, Omvik S, Wedel H: Cardiovascular morbidity and mortality in the losartan intervention for endpoint reduction in hypertension study (LIFE): a randomised trial against atenolol. Lancet 2002, 359:995-1003.

I16. Management Committee on behalf of the High Blood Pressure Research Council of Australia: Australian comparative outcome trial of angiotensin-converting enzyme inhibitor-and diuretic-based treatment of hypertension in the elderly (ANBP2): objectives and protocol. Clin Exp Pharmacol Physiol 1997, 24: $188-192$.

I 17. Wing LMH, Reid CM, Ryan P, Beilin LJ, Brown MA, Jennings GLR, Jonston $\mathrm{Cl}$, McNeil J], Macdonald GJ, Marley JE, TO M, West MJ: A comparison of outcomes with angiotensin-converting-enzyme inhibitors and diuretics for hypertrension in the elderly. $N$ Engl J Med 2003, 348:583-592.

I 18. Black HR, Elliott WJ, Neaton JD, Grandits G, Grambsch P, Grimm RH, Hansson L, Lacourciere Y, Muller J, Sleight P, Weber MA, White WB, Williams G, Wittes J, Zanchetti A, Fakouhi TD: Rationale and design for the controlled ONset Verapamil INvestigation of Cardiovascular Endpoints (CONVINCE) Trial. Control Clin Trials 1998, 19:370-390.

I 19. Black HR, Elliott WJ, Grandits G, Grambsch P, Lucente T, White WB, Neaton JD, Grimm RH, Hansson L, Lacourciere Y, Muller J, Sleight P, Weber MA, Williams G, Wittes J, Zanchetti A, Anders RJ: Principal results of the controlled onset verapamil investigation of cardiovascular end points (CONVINCE) trial. JAMA 2003, 289:2073-2082.

120. Lithell H, Hansson L, Skoog I, Elmfeldt D, Hofman A, Olofsson B, Trenkwalder $P$, Zanchetti $A$ : The study on cognition and prognosis in the elderly (SCOPE): principal results of a randomized double-blind intervention trial. J Hypertens 2003, 2 I :875-886.

121. Yui Y, Sumiyoshi T, Kodama K, Hirayama A, Nonogi H, Kanmatsuse K, Origasa H, limura O, Ishii M, Saruta T, Arakawa K, Hosoda S, Kawai $C$, for the Japanese Multicenter Investigation for Cardiovascular Diseases-B (JMIC-B) Study Group: Comparison of nifedipine retard with angiotensin converting enzyme inhibitors in Japanese hypertensive patients with coronary artery disease: the Japan Multicenter Investigation for Cardiovascular DiseasesB (JMIC-B) Randomized Trial. Hypertens Res 2004, 27: I81-191.

122. Julius S, Kjeldsen SE, Weber M, Brunner HR, Ekman S, Hansson L, Hua T, Laragh J, Mclnnes GT, Mitchell L, Plat F, Schork A, Smith B, Zanchetti $A$, group* VALUE: Outcomes in hypertensive patients at high cardiovascular risk treated with regimens based on valsartan or amlodipine: the VALUE randomised trial. Lancet 2004, 363:2022-2031.

123. Kjeldsen SE, Julius S, Brunner H, Hansson L, Henis M, Ekman S, Laragh J, Mclnnes G, Smith B, Weber M, Zanchetti A: Characteristics of I5,3 I 4 hypertensive patients at high coronary risk. The VALUE trial. The Valsartan Antihypertensive Long-term Use Evaluation. Blood Press 2001, 10:83-91.

124. Mann J, Julius S: The Valsartan Antihypertensive Long-term Use Evaluation (VALUE) trial of cardiovascular events in hypertension. Rationale and design. Blood Press 1998, 7:176-183.

\section{Pre-publication history}

The pre-publication history for this paper can be accessed here: 
http://www.biomedcentral.com/1472-6963/6/47/prepub

Publish with Bio Med Central and every scientist can read your work free of charge

"BioMed Central will be the most significant development for disseminating the results of biomedical research in our lifetime. " Sir Paul Nurse, Cancer Research UK

Your research papers will be:

- available free of charge to the entire biomedical community

- peer reviewed and published immediately upon acceptance

- cited in PubMed and archived on PubMed Central

- yours - you keep the copyright 